\title{
Anionic Hydrogen Cluster Ions as a New Form of Condensed Hydrogen
}

\author{
Michael Renzler, ${ }^{1}$ Martin Kuhn, ${ }^{1}$ Andreas Mauracher, ${ }^{1}$ Albrecht Lindinger, ${ }^{2}$ Paul Scheier, ${ }^{1,}$ and Andrew M. Ellis ${ }^{3, \dagger}$ \\ ${ }^{1}$ Institut für Ionenphysik und Angewandte Physik, Universität Innsbruck, Technikerstrasse 25, A-6020 Innsbruck, Austria \\ ${ }^{2}$ Institut für Experimentalphysik, Freie Universität Berlin, Arnimallee 14, 14195 Berlin, Germany \\ ${ }^{3}$ Department of Chemistry, University of Leicester, University Road, Leicester LE1 7RH, United Kingdom
}

(Received 5 September 2016; published 27 December 2016)

\begin{abstract}
We report the first experimental observation of negatively charged hydrogen and deuterium cluster ions, $\mathrm{H}_{n}^{-}$and $\mathrm{D}_{n}^{-}$, where $n \geq 5$. These anions are formed by an electron addition to liquid helium nanodroplets doped with molecular hydrogen or deuterium. The ions are stable for at least the lifetime of the experiment, which is several tens of microseconds. Only anions with odd values of $n$ are detected, and some specific ions show anomalously high abundances. The sizes of these "magic number" ions suggest an icosahedral framework of $\mathrm{H}_{2}\left(\mathrm{D}_{2}\right)$ molecules in solvent shells around a central $\mathrm{H}^{-}\left(\mathrm{D}^{-}\right)$ion. The first three shells, which contain a total of $44 \mathrm{H}_{2}$ or $\mathrm{D}_{2}$ molecules, appear to be solidlike, but thereafter a more liquidlike arrangement of the $\mathrm{H}_{2}\left(\mathrm{D}_{2}\right)$ molecules is adopted.
\end{abstract}

DOI: 10.1103/PhysRevLett.117.273001

The simplest polyatomic anion is $\mathrm{H}_{3}^{-}$. Theoretical studies of this ion go back as far as 1937 [1], but it is only through relatively recent high-quality electronic structure calculations that this ion has been shown to be stable [2-4]. The latest calculations reveal a shallow potential energy well which can contain a small number of bound vibrational levels. The dissociation energy from the zero point level of $\mathrm{H}_{3}^{-}$is calculated to be only $\sim 70 \mathrm{~cm}^{-1}$ [4]. The $\mathrm{H}_{3}^{-}$ground state is mainly electronically bound, whereas the first excited state of $\mathrm{H}_{3}^{-}$is quasistationary [5]. In some regions of the nuclear coordinate space, these two states are coupled nonadiabatically.

Experimental studies of $\mathrm{H}_{3}^{-}$have proved challenging. Although the detection of this ion was first reported more than 40 years ago [6,7], the signals were weak, leading to questions about the reliability of the claims [8]. It is only more recently, via experiments in dielectric discharge plasmas, that $\mathrm{H}_{3}^{-}$has been observed at an abundance that made firm identification possible [3]. Weak signals from $\mathrm{H}_{3}^{-}$and $\mathrm{D}_{3}^{-}$have also been detected in secondary-ion mass spectrometry experiments by the sputtering of solid $\mathrm{HfH}_{2}$ and $\mathrm{TiD}_{2}$ targets [9].

$\mathrm{H}_{3}^{-}$is the smallest member of the group of polyatomic $\mathrm{H}_{n}^{-}$cluster anions containing an odd number of hydrogen atoms. The first theoretical study for ions with $n \geq 5$ was carried out by Sapse et al. [10], who were partly motivated by the possibility that these ions might be present in the interstellar medium. Sapse et al. performed Hartree-Fock calculations with a small basis set. The largest cluster considered was $\mathrm{H}_{13}^{-}$, and this and all smaller clusters were found to adopt highly symmetric arrangements of $\mathrm{H}_{2}$ around a central $\mathrm{H}^{-}$ion. Thus, $\mathrm{H}_{7}^{-}, \mathrm{H}_{9}^{-}, \mathrm{H}_{11}^{-}$, and $\mathrm{H}_{13}^{-}$ showed planar trigonal, tetrahedral, pentagonal bipyramidal, and octahedral arrangements of $\mathrm{H}_{2}$ molecules around the $\mathrm{H}^{-}$ion, respectively, whereas $\mathrm{H}_{5}^{-}$was calculated to be linear. In a subsequent study, Hirao and Yamabe [11] employed the configuration interaction method and obtained similar cluster structures to Sapse et al. However, in more recent calculations, Huang and Matta have combined density functional theory with harmonic vibrational frequency calculations and found that the highly symmetric structures reported previously were not true minima on their potential energy surfaces [12]. For example, Huang and Matta determined $\mathrm{H}_{5}^{-}$to be strongly bent rather than linear. Consequently, there are real doubts about the basic structures of $\mathrm{H}_{n}^{-}$ions that need to be resolved, and given that there are no reliable estimates of the actual dissociation energies $\left(D_{0}\right)$ of these clusters, it is not even clear if anions with $n>3$ are stable.

Here we report the first experimental detection of anionic hydrogen and deuterium clusters larger than $\mathrm{H}_{3}^{-} / \mathrm{D}_{3}^{-}$and have done so for a wide range of cluster sizes. The experimental procedure involved the formation of the corresponding neutral $\left(\mathrm{H}_{2}\right)_{N}$ and $\left(\mathrm{D}_{2}\right)_{N}$ clusters by adding $\mathrm{H}_{2}$ or $\mathrm{D}_{2}$ gas to liquid helium nanodroplets. The neutral clusters were cooled to the ambient temperature of the helium nanodroplets, $0.38 \mathrm{~K}$ [13], prior to an impact by a beam of electrons with a controlled energy. $\mathrm{H}_{2}$ and $\mathrm{D}_{2}$ are heliophilic (have a negative chemical potential when immersed in liquid helium [13]) and so will reside inside the helium droplets rather than on the surface. The helium droplets were then exposed to a beam of electrons, which generated anionic products in the gas phase that were detected by mass spectrometry. The transfer of negative charge to the $\left(\mathrm{H}_{2}\right)_{n}$ and $\left(\mathrm{D}_{2}\right)_{N}$ clusters occurs via a mobile electron bubble, whose formation has a threshold energy in excess of $1 \mathrm{eV}$ in order to inject the electron into the helium conduction band [14]. The droplets used in the present work were relatively large ( $\sim 10^{6}$ helium atoms), and for droplets of this size the electron bubble, although 


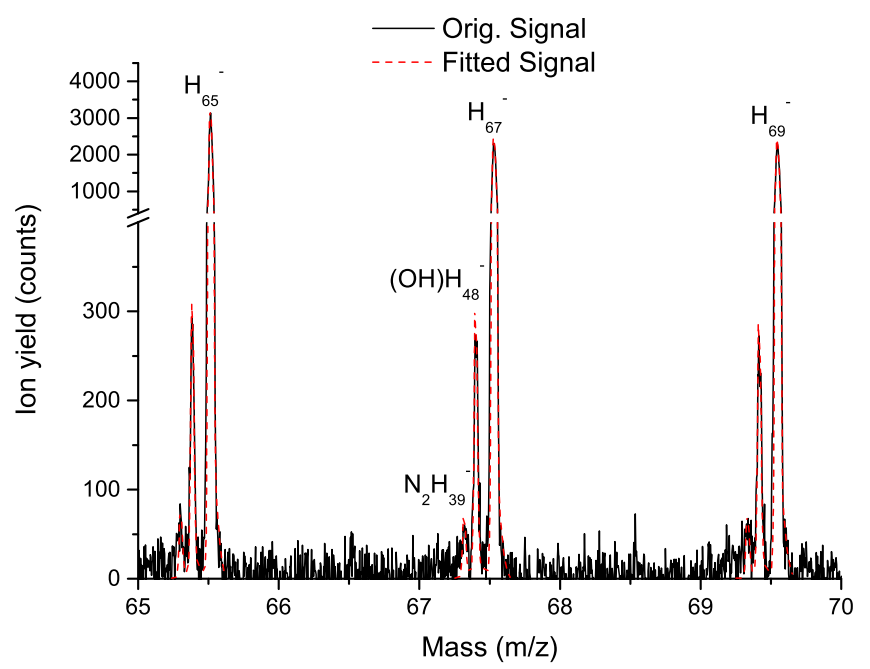

FIG. 1. Section of the mass spectrum recorded for hydrogen cluster anions. The dominant series of ions seen here are from $\mathrm{H}_{n}^{-}$, but small quantities of $\left(\mathrm{H}_{n}\right) \mathrm{OH}^{-}$and $\left(\mathrm{H}_{n}\right) \mathrm{N}_{2}^{-}$are also detected because of trace quantities of water and air in the apparatus. Also shown is a simulation derived from IsotopeFit [24], which is used to deconvolute overlapping contributions from different sized clusters and from different isotope combinations. The agreement between the fitted and experimental data is essentially perfect in this region.

metastable, is relatively long-lived and the electron can find and transfer onto the molecular cluster [15].

The anions detected by mass spectrometry consisted of bare $\mathrm{H}_{n}^{-}$and $\mathrm{D}_{n}^{-}$ions, as seen in the representative mass spectrum for $\mathrm{H}_{n}^{-}$shown in Fig. 1. In Figs. 2(a) and 2(b), we show how the abundances of $\mathrm{H}_{n}^{-}$and $\mathrm{D}_{n}^{-}$vary as a function of $n$ following the addition of electrons at a kinetic energy of $11 \mathrm{eV}$ (the dependence of the cluster ion production on electron energy will be discussed in a subsequent publication). Cluster ions can be observed with $n$ extending well beyond 100. Crucially, only anions with odd $n$ are seen, suggesting that these are produced by dissociative electron attachment to an $\mathrm{H}_{2}$ or $\mathrm{D}_{2}$ molecule, which is a well-known process in the gas phase [16-18]. Having formed $\mathrm{H}^{-}$, this can then combine with the other $\mathrm{H}_{2}$ molecules in the cluster either by a three-body collision association [3] or via radiative association $[4,19]$. The latter might lead to the formation of $\mathrm{H}_{3}^{-}$anions in the interstellar medium, where a third body is not available, but in a helium droplet a threebody collisional association mechanism is far more likely. The net effect is the formation of a cluster anion via the process

$$
\left(\mathrm{H}_{2}\right)_{N}+e^{-} \rightarrow \mathrm{H}_{n}^{-}+\mathrm{H},
$$

where $n=2 N-1$. Also possible, but not shown in reaction (1), is the loss of one or more intact $\mathrm{H}_{2}$ molecules from the cluster as the anion is formed. The observation of only odd $n$ anions shows that the surrounding helium is

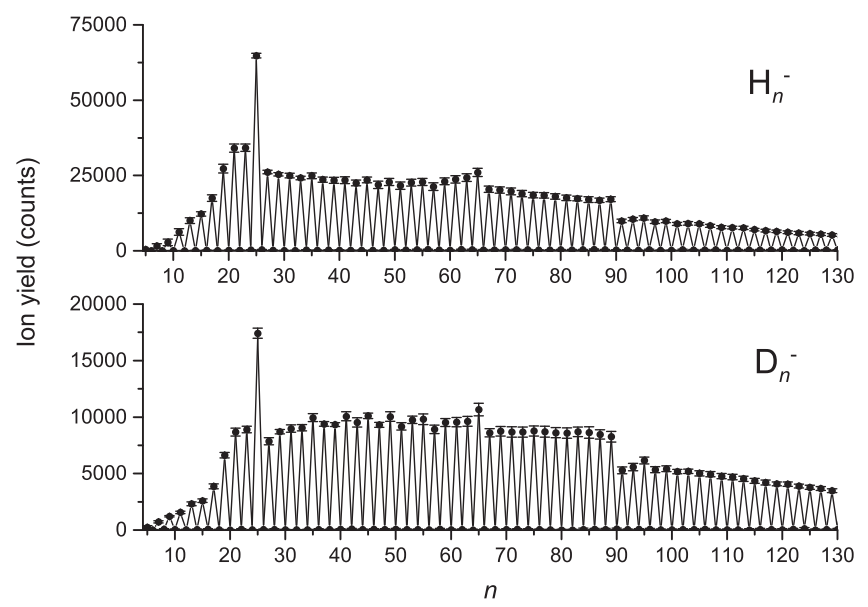

FIG. 2. Plots of the measured ion signal levels for (a) $\mathrm{H}_{n}^{-}$and (b) $\mathrm{D}_{n}^{-}$as a function of $n$. The signal levels are expressed as integrated ion counts for a given mass spectral peak.

unable to prevent dissociation of the initially formed transient anion. This is in marked contrast to helium droplet experiments focusing on the cationic clusters, $\mathrm{H}_{n}^{+}$, formed by electron ionization. Although odd $n$ ions overwhelmingly dominate in the gas phase [20], even $n$ ions become significant positively charged products when experiments are carried out in helium nanodroplets [21]. This is presumably because of partial quenching of the initially "hot" ions by the surrounding helium, which thereby stabilizes the even $n$ ions. The absence of even $n$ clusters for the anions is consistent with the very short lifetimes expected for $\mathrm{H}_{2}^{-}$and $\mathrm{D}_{2}^{-}$(on the order of $10^{-15} \mathrm{~s}$ ) following an electron attachment to the cold neutral molecule [22,23], although the lifetimes extend into the microsecond regime when the anions are highly rotationally excited [23].

Particularly striking in Fig. 2 are anomalously intense signals at $n=25,65$, and 89 , which are seen equally clearly for hydrogen and deuterium. We associate each of these peaks with cluster ions of increased stability relative to the cluster ions with one additional $\mathrm{H}_{2}$ or $\mathrm{D}_{2}$ molecule. These "magic number" ions correspond to 12,32 , and 44 $\mathrm{H}_{2}\left(\mathrm{D}_{2}\right)$ molecules in combination with a hydride (deuteride) anion. In what follows, we explicitly mention only hydrogen, but the same general conclusions also apply to deuterium. The smallest magic anion is consistent with an icosahedral arrangement of $12 \mathrm{H}_{2}$ molecules in the first shell around a central $\mathrm{H}^{-}$core. The larger magic cluster anions can also be interpreted in terms of icosahedral-like structures by analogy with cationic clusters between atomic ions and helium atoms [25]. Thus, the $n=65$ magic anion can be explained by a second shell of $20 \mathrm{H}_{2}$ molecules occupying the 20 triangular faces of a regular icosahedron, generating a dodecahedral arrangement. This is a layer often referred to as an anti-Mackay structure [26,27]. For the $n=89$ anion, there are a further $12 \mathrm{H}_{2}$ molecules 


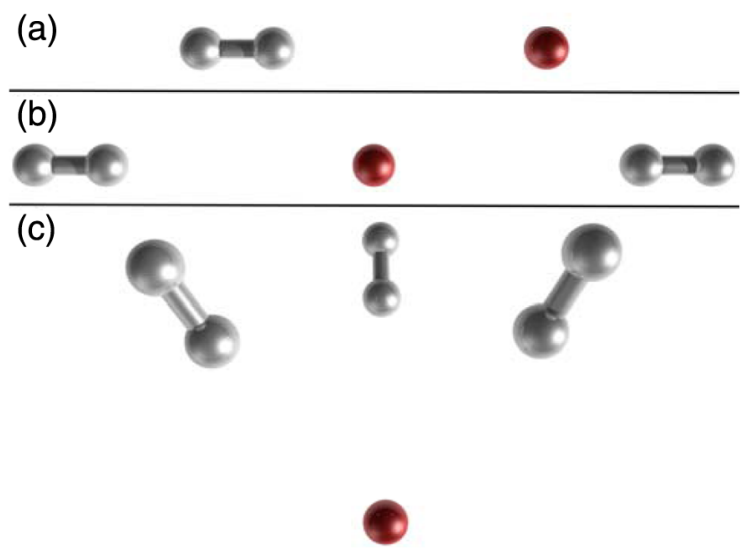

FIG. 3. Calculated equilibrium structures for $\mathrm{H}_{3}^{-}, \mathrm{H}_{5}^{-}$, and $\mathrm{H}_{7}^{-}$, which are found to be linear for $\mathrm{H}_{3}^{-}$and $\mathrm{H}_{5}^{-}$and trigonal pyramidal for $\mathrm{H}_{7}^{-}\left(\mathrm{C}_{3 \mathrm{v}}\right.$ point group symmetry). The gray spheres indicate the positions of the nuclei in the hydrogen molecules, whereas the red sphere indicates the position of the nucleus of the atomic hydrogen anion. Detailed information about the structures, including those of the neutral clusters, can be found in Supplemental Material [30], along with other information such as the charge density distributions in the highest occupied molecular orbitals of the anions.

occupying the vertices of the inner shell icosahedron, giving a rhombic tricontahedron structure. This effectively completes a well-defined third solvation shell.

Despite the weak ion-induced dipole binding expected in these anions and the quantum effects that might be derived from the zero point vibrational energy given the light atoms, the observed magic numbers indicate solidlike shell structures that extend out to three complete shells. Bartl et al. [25] have recently reported equivalent shells for $\mathrm{He}_{n} \mathrm{Ar}^{+}$and draw an analogy between icosahedral $\mathrm{He}_{12} \mathrm{Ar}^{+}$and $\mathrm{C}_{60}^{+}$, where the coating of the latter by helium leads to a rigid helium layer [28]. It is the corrugations on the $\mathrm{C}_{60}^{+}$surface that lead to a rigid helium layer, and the same applies to the helium coating of $\mathrm{He}_{12} \mathrm{Ar}^{+}$. $\left(\mathrm{H}_{2}\right)_{12} \mathrm{H}^{-}$ presumably behaves in a similar way, and the corrugations on the surface of the cluster may even be enhanced by the anisotropy of the $\mathrm{H}_{2}$ molecules. Having completed the first three shells around the hydride ion, the addition of further $\mathrm{H}_{2}$ molecules leads to no other observable magic number features, suggesting that $\mathrm{H}_{2}$ molecules beyond the third solvation shell have a more liquidlike arrangement.

We can compare our findings for anions with magic number data extracted from a Raman spectroscopic study of neutral $\left(\mathrm{H}_{2}\right)_{N}$ clusters in a free jet expansion [29]. Very weak intermolecular forces are expected between the hydrogen molecules in $\left(\mathrm{H}_{2}\right)_{N}$ clusters, and yet the Raman data seem to imply approximate magic numbers through maxima in the signal levels. These are broad maxima as a function of the cluster size, rather than the sharp features seen in the current study, but they nevertheless seemed to peak near $N=13$ and 33, with tentative evidence for a third magic
TABLE I. Calculated adiabatic and vertical electron affinities of $\mathrm{H}, \mathrm{H}_{3}, \mathrm{H}_{5}$, and $\mathrm{H}_{7}$.

\begin{tabular}{lcc}
\hline \hline Species & AEA/eV & VEA/eV \\
\hline $\mathrm{H}^{\mathrm{a}}$ & 0.74 & \\
$\mathrm{H}_{3}$ & 0.78 & 0.77 \\
$\mathrm{H}_{5}$ & 0.81 & 0.81 \\
$\mathrm{H}_{7}$ & 0.87 & 0.69 \\
\hline \hline
\end{tabular}

${ }^{a}$ The experimental value for the electron affinity of atomic hydrogen is $0.75420 \pm 0.0002 \mathrm{eV}$ [32].

number near 55. Again, the suggestion is that semirigid structures might form for $\left(\mathrm{H}_{2}\right)_{N}$ clusters, and for the first two shells the magic numbers match our findings for the anions. A magic number of 55 would correspond to the completion of the second layer of an icosahedral structure, but we see no evidence for such a structure in the case of the cluster anions.

The $\mathrm{H}_{n}^{-}$and $\mathrm{D}_{n}^{-}$must be stable for at least tens of microseconds in order to reach the detector in our experiments. This is consistent with the observations made by Wang et al. for $\mathrm{H}_{3}^{-}$, whose lifetime was found to be $>8 \mu \mathrm{s}$ [3]. The stabilities of these anions must be a reflection of the significant electron affinities of the corresponding neutral clusters. To explore this, we have performed $a b$ initio calculations on the three smallest neutral clusters, $\mathrm{H}_{3}, \mathrm{H}_{5}$, and $\mathrm{H}_{7}$, and the corresponding anions. Calculations were performed using the MP2 method for full geometry optimization and the CCSD method for the evaluation of the total electronic energies: In both cases, large Gaussian basis sets of quadruple zeta quality were employed, as detailed in Supplemental Material [30,31]. Structures found for the anions are shown in Fig. 3. A number of conclusions can be reached from these calculations. First, $\mathrm{H}_{5}^{-}$is found to be linear, which is different from a recent DFT study of small $\mathrm{H}_{n}^{+}$ions [12] but in good agreement with earlier $a b$ initio studies of these ions $[10,11]$. In contrast, we find a trigonal pyramidal structure for $\mathrm{H}_{7}^{-}$, which agrees with the aforementioned DFT study and disagrees with the planar trigonal structures predicted in the earlier theoretical work. Second, a simple Mulliken analysis shows that the negative charge is mainly on a single hydrogen atom in all three cluster anions. Finally, we have determined electron affinities of $\mathrm{H}_{3}, \mathrm{H}_{5}$, and $\mathrm{H}_{7}$ and summarize the findings in Table I. Also shown is the electron affinity of the $\mathrm{H}$ atom, which is well known experimentally and provides a confirmation of the good quality of the calculations. The calculated electron affinities of $\mathrm{H}_{3}, \mathrm{H}_{5}$, and $\mathrm{H}_{7}$ are comparable and significant, suggesting that the corresponding anions would be stable against autodetachment.

In summary, we have made the first observation of $\mathrm{H}_{n}^{-}$ and $\mathrm{D}_{n}^{-}$cluster ions with $n \geq 5$. These anions, which are held together by weak induction forces, can be seen across a wide range of sizes and extend to well beyond $n=100$ when formed in helium nanodroplets. Furthermore, magic numbers reveal symmetrical, rigid structures for the clusters 
extending out to three solvation shells. These experimental observations now open the way to further studies of these rather fundamental cluster anions. Among the things in need of further investigation are the structures of these ions and an assessment of the role of any quantum effects, such as vibrational delocalization and bosonic exchange, on their properties.

This work was given financial support by the Austrian Science Fund (FWF) Wien (P26635 and I978).

*Paul.Scheier@uibk.ac.at †andrew.ellis@le.ac.uk

[1] D. Stevenson and J. Hirschfelder, J. Chem. Phys. 5, 933 (1937).

[2] J. Stärk and W. Meyer, Chem. Phys. 176, 83 (1993).

[3] W. Wang, A. K. Belyaev, Y. Xu, A. Zhu, C. Xiao, and X.-F. Yang, Chem. Phys. Lett. 377, 512 (2003).

[4] M. Ayoux, O. Dulieu, R. Guérout, J. Robert, and V. Kokoouline, J. Chem. Phys. 132, 194309 (2010).

[5] A. K. Belyaev, A.S. Tiukanov, and W. Domcke, Chem. Phys. 325, 378 (2006).

[6] R. E. Hurley, Nucl. Instrum. Methods 118, 307 (1974).

[7] W. Aberth, R. Schnitzer, and M. Anbar, Phys. Rev. Lett. 34, 1600 (1975).

[8] Y. K. Bae, M. J. Coggiola, and J. R. Peterson, Phys. Rev. A 29, 2888 (1984).

[9] H. Gnaser and R. Golser, Phys. Rev. A 73, 021202 (2006).

[10] A. M. Sapse, M. T. Rayez-Meaume, J. C. Rayez, and L. J. Massa, Nature (London) 278, 332 (1979).

[11] K. Hirao and S. Yamabe, Chem. Phys. 80, 237 (1983).

[12] L. Huang and C. F Matta, J. Phys. Chem. A 115, 12445 (2011).

[13] J. P. Toennies and A. F. Vilesov, Angew. Chem., Int. Ed. 43, 2622 (2004).

[14] U. Henne and J. P. Toennies, J. Chem. Phys. 108, 9327 (1998).
[15] M. Rosenblit and J. Jortner, J. Chem. Phys. 124, 194506 (2006).

[16] M. Allan and S. F. Wong, Phys. Rev. Lett. 41, 1791 (1978).

[17] H. Drexel, G. Senn, T. Fiegele, P. Scheier, A. Stamatovic, N. J. Mason, and T. D. Märk, J. Phys. B 34, 1415 (2001).

[18] E. Krishnakumar, S. Denifl, I. Čadež, S. Markelj, and N. J. Mason, Phys. Rev. Lett. 106, 243201 (2011).

[19] M. Ayouz, R. Lopes, M. Raoult, O. Dulieu, and V. Kokoouline, Phys. Rev. A 83, 052712 (2011).

[20] R. Clampitt and L. Gowland, Nature (London) 223, 815 (1969).

[21] S. Jaksch, A. Mauracher, A. Bacher, S. Denifl, F. Ferreira da Silva, H. Schöbel, O. Echt, T. D. Märk, M. Probst, D. K. Bohme, and P. Scheier, J. Chem. Phys. 129, 224306 (2008).

[22] M. Č́ížek, J. Horáček, and W. Domcke, J. Phys. B 31, 2571 (1998).

[23] R. Golser, H. Gnaser, W. Kutschera, A. Priller, P. Steier, A. Wallner, M. Č́žzek, J. Horáček, and W. Domcke, Phys. Rev. Lett. 94, 223003 (2005).

[24] S. Ralser, J. Postler, M. Harnisch, A. M. Ellis, and P. Scheier, Int. J. Mass Spectrom. 379, 194 (2015).

[25] P. Bartl, C. Leidlmair, S. Denifl, P. Scheier, and O. Echt, J. Phys. Chem. A 118, 8050 (2014).

[26] M. R. Hoare, Adv. Chem. Phys. 40, 49 (1979).

[27] J. P. K. Doye, D. J. Wales, and R. S. Berry, J. Chem. Phys. 103, 4234 (1995).

[28] C. Leidlmair, Y. Wang, P. Bartl, H. Schöbel, S. Denifl, M. Probst, M. Alcamí, F. Martin, H. Zettergren, K. Hansen, O. Echt, and P. Scheier, Phys. Rev. Lett. 108, 076101 (2012).

[29] G. Tejeda, J. M. Fernández, S. Montero, D. Blume, and J. P. Toennies, Phys. Rev. Lett. 92, 223401 (2004).

[30] See Supplemental Material at http://link.aps.org/ supplemental/10.1103/PhysRevLett.117.273001 for further information on the ab initio calculations, which includes Ref. [31].

[31] Gaussian 09, Revision D.01, M. J. Frisch et al., Gaussian, Inc., Wallingford, CT, 2009.

[32] K. R. Lykke, K. K. Murray, and W. C. Lineberger, Phys. Rev. A 43, 6104 (1991). 